\title{
A plasmonic biosensor array by block copolymer lithography $\dagger$
}

\author{
Dong Ok Shin, ${ }^{a}$ Jong-Ryul Jeong, ${ }^{b}$ Tae Hee Han, ${ }^{a}$ Chong Min Koo, ${ }^{c}$ Hye-Jeong Park, ${ }^{d}$ Yong Taik Lim*e \\ and Sang Ouk Kim*a
}

\author{
Received 4th May 2010, Accepted 5th June 2010 \\ DOI: $10.1039 / \mathrm{c0jm01319f}$
}

\begin{abstract}
Highly uniform and dense, hexagonal noble metal nanoparticle arrays were achieved on large-area transparent glass substrates via scalable, parallel processing of block copolymer lithography. Exploring their localized surface plasmon resonance (LSPR) characteristics revealed that the Ag nanoparticle array displayed a UV-vis absorbance spectrum sufficiently narrow and intense for biosensing application. A highly-sensitive, label-free detection of prostate cancer specific antibody (anti-PSA) with sub-ng ml ${ }^{-1}$ level detection limit $\left(0.1 \sim 1 \mathrm{ng} \mathrm{ml}^{-1}\right)$ has been accomplished with the plasmonic nanostructure. Our approach offers a valuable route to a low-cost, manufacture-scale production of plasmonic nanostructures, potentially useful for various photonic and optoelectronic devices.
\end{abstract}

\section{Introduction}

The extraordinary optical properties of noble metal (Ag, Au, Pt) nanostructures have been exploited for a broad range of applications such as plasmonic waveguides, ${ }^{1-3}$ surface-enhanced raman scattering (SERS), ${ }^{4-6}$ and real-time chemical/biological sensors. ${ }^{7-11}$ These metal nanostructures exhibit an intense UV-vis absorption band, which is absent in bulk, providing that an incident photon frequency is resonant with the collective oscillation of free electrons. As a result, the local electromagnetic fields are remarkably enhanced, and generally known as localized surface plasmon resonance (LSPR). ${ }^{12,13}$ The characteristic wavelength and intensity of the LSPR spectra are extremely sensitive to the shape, size, interparticle distance, and dielectric environment of the particles, offering a robust platform for refractive index based sensing devices. ${ }^{12,14}$

Various nanopatterning methods such as electron beam lithography or nanoimprint lithography (NIL) have been employed to create plasmonic metal nanostructures. Nevertheless, low throughput serial processing or technical hardship arising from direct mechanical contact does not allow for a mass-producible process. ${ }^{15-17}$ An alternative method is selfassembly, where the spontaneous organization of nanoscale building blocks allows for the large-scale, parallel production of

${ }^{a}$ Department of Materials Science and Engineering, KAIST, Daejeon, 305-701, Republic of Korea. E-mail: sangouk.kim@kaist.ac.kr; Fax: +82 42350 3310; Tel: +82423503339

${ }^{b}$ Department of Materials Science and Engineering and Graduate School of Green Energy Technology, Chungnam National University, Daejeon, 305764, Republic of Korea

'Polymer Hybrids Center, Korea Institute of Science and Technology (KIST), P.O. BOX 131, Cheongryang, Seoul, Republic of Korea

${ }^{d}$ OLED Product Development Team, Samsung Mobile Display, 508, Sunsung-Dong, Seobuk-gu, Cheonan City, Chungcheong nam-do, Republic of Korea

${ }^{e}$ Graduate School of Analytical Science and Technology, Chungnam National University, Daejeon, 305-764, Republic of Korea. E-mail: yongtaik@cnu.ac.kr

$\uparrow$ Electronic supplementary information (ESI) available: Simulation of LSPR with a three dimensional finite-difference time-domain (FDTD) calculation, additional UV-vis absorbance data. See DOI: 10.1039/c0jm01319f periodic nanostructures. A representative example is nanosphere lithography (NSL), which utilizes a self-assembled hexagonal nanosphere array as a lithographic mask. The metal nanostructures generated by NSL have been successfully implemented for a new class of LSPR devices having hundreds of nanometres scale characteristic dimensions. ${ }^{10,18}$ Another emerging self-assembly approach is block copolymer lithography. A variety of periodic nanoscale morphologies such as sphere or cylinder array, or lamellae self-assembled in block copolymer thin films enable ultrafine and dense nanopatterning with the characteristic dimension of a $5 \sim 50 \mathrm{~nm}$ scale. ${ }^{19-28}$ The scalable and parallel assembly of block copolymers enables large-scale nanopatterning with fine tunability over the shape, density, and characteristic dimensions. Despite its enormous advantages, block copolymer lithography has thus far mostly been employed for the fabrication of electronic or magnetic nanostructures. ${ }^{29,30}$

In this work, we present a facile and robust, manufacture-scale approach to create highly dense and uniform nanoscale plasmonic arrays by block copolymer lithography. Recently, we demonstrated that block copolymer lithography is a highly efficient nanolithography for a broad spectrum of target substrates including metals, ceramics, semiconductors, and polymers. ${ }^{31}$ Here we extended block copolymer lithography to an optically transparent pyrex glass substrate for spectroscopic assay that combines highly specific biomolecular interactions with plasmonic resonance effects. The optically transparent substrate is crucial for the high-throughput spectroscopic detection of the LSPR absorption band. Block copolymer lithography generated uniform hexagonal noble metal nanoparticle arrays on glass substrates over an arbitrarily large area. We explored their LSPR characteristics and found that an Ag nanoparticle array displayed a UV-vis absorbance spectrum sufficiently narrow and intense for biosensing applications. The significant influence of biomolecular interactions on the LSPR peak wavelength (LSPR $\left.\lambda_{\max }\right)$ was investigated while the responses were attributed to the specific binding events between antigen and antibody. Highly-sensitive, label-free detection of prostate specific antibodies (anti-PSA) with a sub-ng $\mathrm{ml}^{-1}$ level detection limit has been demonstrated. ${ }^{32,33}$ 


\section{Experimental}

\section{Materials}

Polystyrene-block-poly(methyl methacrylate) (PS-b-PMMA) (SM1: $M_{n}$ of PS block: $140 \mathrm{~kg} \mathrm{~mol}^{-1}, M_{n}$ of PMMA block: $60 \mathrm{~kg}$ mol ${ }^{-1}$, SM2: $M_{n}$ of PS block: $5 \mathrm{~kg} \mathrm{~mol}^{-1}, M_{n}$ of PMMA block: 5 $\mathrm{kg} \mathrm{mol}^{-1}$ ) was purchased from Polymer Source. Thermal evaporation sources for gold $(99.999 \%)$, silver (99.99\%), copper (99.997\%) and platinum sputtering targets were purchased from Tasco. 11-mercaptoundecanoic acid (11-MUA) and 1-octanethiol (1-OT) were purchased from Aldrich. 1-ethyl-3-[3-dimethylaminopropyl]carbodiimide hydrochloride (EDC) was purchased from Pierce. Trizma base and calcium chloride $\left(\mathrm{CaCl}_{2}\right)$ dihydrate were purchased from Sigma. Sodium chloride ( $\mathrm{NaCl}$ ) was purchased from Junsei. Phosphate-buffered saline (PBS) solutions of $10 \mathrm{nM}$ and $\mathrm{pH} 7.4$ were purchased from Invitrogen. Kallikrein 3, commonly known as prostate specific antigen (PSA) was purchased from R\&D Systems, Inc., and antiPSA (mouse monoclonal antibody) was purchased from Santa Cruz Biotechnology, Inc. Anti C-reactive protein was purchased from Calbiochem.

\section{Plasmonic biosensor array chip preparation by block copolymer lithography}

The surface of a 5 inch pyrex glass substrate was chemically cleaned and modified with a random brush copolymer as describe elsewhere. ${ }^{31,34}$ A thin film (thickness: $75 \mathrm{~nm}$ ) of block copolymer blends, consisting of 10:4 mixture of high molecular weight SM1 and low molecular weight SM2, was spin-coated on the neutrally modified glass substrate. Then with high temperature annealing at $200{ }^{\circ} \mathrm{C}$, the block copolymer blends film spontaneously assembled into a nanoscale morphology comprised of hexagonal vertical PMMA cylinders in a PS matrix. The block copolymer film was irradiated with UV and subsequently rinsed with acetic acid and water to selectively remove the PMMA cylinder cores and crosslink the remaining PS matrix. After template preparation, the 5-inch pyrex substrate was diced into $1.6 \times 1.6 \mathrm{~cm}^{2}$ chip size. Noble metal ( $\mathrm{Au}, \mathrm{Ag}, \mathrm{Cu}, \mathrm{Pt}$ ) films were deposited over the hexagonal nanoporous block copolymer template. After metal deposition, the remnant PS nanoporous template was lifted off by ultrasonication in toluene. A highly uniform hexagonal metal nanoparticle array replicating the block copolymer template morphology remained at the transparent pyrex glass substrate.

\section{Surface chemistry for antibody (anti-PSA) detection}

The prepared plasmonic array was functionalized with the selfassembled monolayer (SAM) mixture (mixture of $3 \mathrm{~mol}$ of $1 \mathrm{mM}$ 1-octanethiol (1-OT) and $1 \mathrm{~mol}$ of $1 \mathrm{mM}$ 11-mercaptoundecanoic acid (11-MUA)) by incubation in an ethanol solution for 12 24 h. After SAM modification, the plasmonic array was thoroughly rinsed with ethanol and dried with $\mathrm{N}_{2}$. The sensory array was incubated in $1 \mu \mathrm{g} \mathrm{ml}^{-1}$ of PSA in TCN solution $(0.15 \mathrm{M} \mathrm{NaCl}, 10$ $\mathrm{mM} \mathrm{CaCl}_{2}$ ) (pH 7.5) with $20 \mu \mathrm{g} \mathrm{ml}^{-1}$ of EDC coupling agent for $1 \mathrm{~h}$ to induce amide bonding between the amine groups in the PSA and the carboxyl groups on the SAM terminated metal nanoparticle array. Finally, the plasmonic array was incubated for $1 \mathrm{~h}$ in anti-PSA solution (diluted to various concentrations in $10 \mathrm{mM}$ PBS for antibody detection), which was followed by washing with PBS and drying with $\mathrm{N}_{2}$.

\section{Characterization}

The UV-vis absorbance spectrum of plasmonic arrays was measured using a UV-3101 PC spectrophotometer (Shimadzu Co., Japan). The nanoscale morphology of the block copolymer thin films and the created metal nanoparticle arrays were imaged using a Hitachi S-4800 SEM. The surface morphology of the metal nanoparticle arrays was analyzed using AFM (Veeco, USA) in tapping mode.

\section{Results and discussion}

A viable fabrication route to LSPR nanostructures requires low-cost mass-production of a periodic metal nanostructure with a proper structural dimension. We developed a 5 inch wafer scale block copolymer lithography process to ensure the mass-producibility.

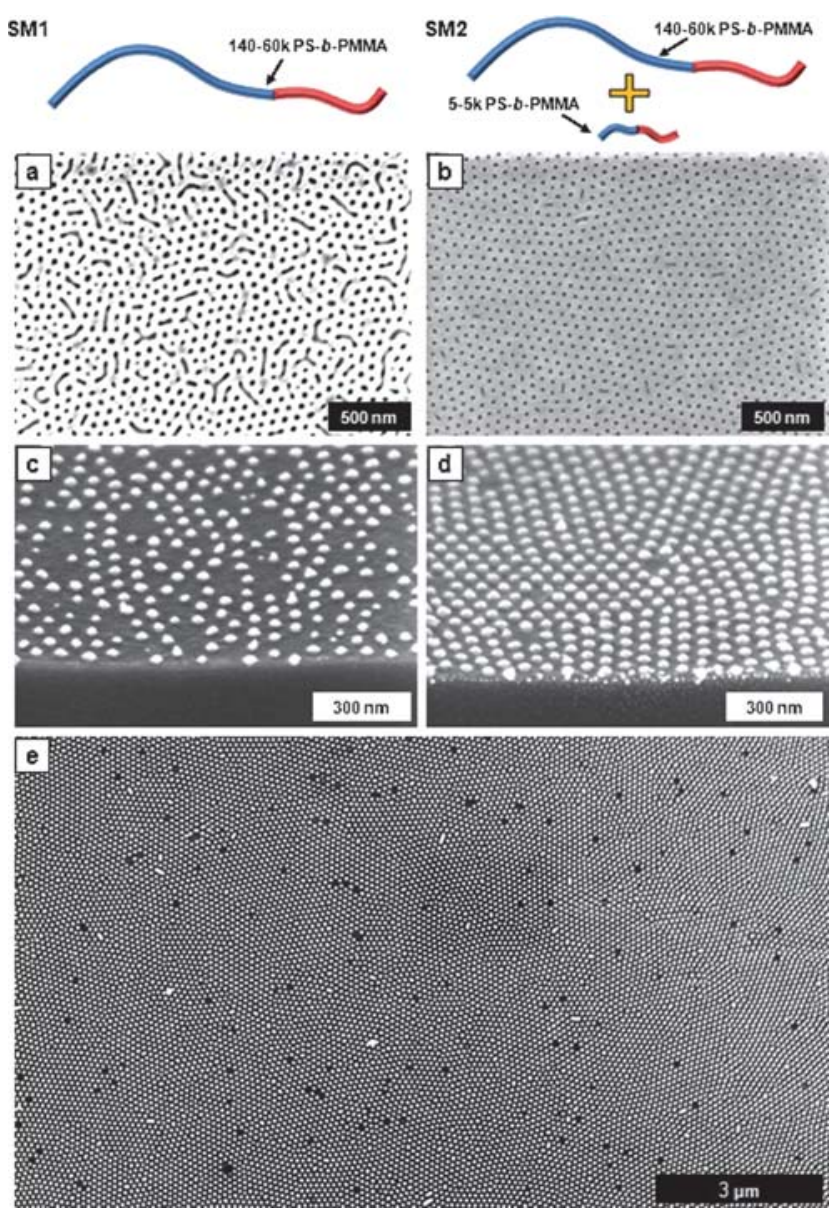

Fig. 1 SEM images of a block copolymer film and nanoparticle array derived from the pure SM1 and the blends of SM1 \& SM2. The lateral ordering of nanodomain was poor (a) in pure SM1 film, while it was significantly enhanced (b) in the blends film, (c) and (d) clearly compare the resulting nanoparticle array morphology from (a) and (b), respectively. (e) A broad field SEM image of the $\mathrm{Ag}$ nanoparticle array prepared from (b). 
The desired structural dimension of the plasmonic array attainable by block copolymer lithography was evaluated by a simulation of LSPR UV-vis absorption behavior. (see ESI, $\uparrow$ Fig. S1) A hexagonal Ag nanoparticle array with a mean diameter of 40 $\mathrm{nm}$ and center-to-center distance between neighbours of $80 \mathrm{~nm}$ was found to exhibit a strong absorption band located in the visible wavelength range. We used a high molecular weight asymmetric polystyrene-block-poly(methyl methacrylate) (PS- $b$ PMMA, SM1; $M_{n}$ of PS block: $140 \mathrm{~kg} \mathrm{~mol}^{-1}, M_{n}$ of PMMA block: $60 \mathrm{~kg} \mathrm{~mol}^{-1}$ ) to achieve this desired dimension. However, the lateral ordering of self-assembled morphology of this polymer was significantly poor due to the slow assembly of the large molecular weight block copolymers (Fig. 1(a) and 1(c)). ${ }^{35}$ To promote self-assembly, a minor part of the low molecular weight PS- $b$-PMMA (SM2; $M_{n}$ of PS \& PMMA blocks: $5 \mathrm{~kg} \mathrm{~mol}^{-1}$ ) was blended with SM1. This low molecular weight SM2 with a low value of $\chi N$ was homogeneously distributed throughout a block copolymer film without any preference for PS or PMMA components. As a consequence, it greatly enhanced the mobility and, thus, the self-assembly of high molecular weight SM1 without significantly influencing the structural dimension of the self-assembled morphology. ${ }^{36}$ The lateral ordering and uniformity of self-assembled morphology could be remarkably enhanced in the blends film with the SM2/SM1 weight ratio of 0.4 (Fig. 1(b)). The resulting nanoparticle array produced by selective metal deposition and subsequent lift-off also revealed a greatly enhanced uniformity over an arbitrarily large substrate area (Fig. 1(d) and 1(e)).

Fig. 2 schematically describes the overall process for a plasmonic biosensor array. A 5 inch pyrex glass substrate was chemically modified with a neutral $\mathrm{P}(\mathrm{S}-r$-MMA) random block copolymer brush to have an identical surface tension to the PS and PMMA components. A PS- $b$-PMMA block copolymer blends film was spin-coated on the neutrally modified pyrex glass substrate and thermally annealed at $200{ }^{\circ} \mathrm{C}$ to form a block copolymer template with a hexagonal vertical cylinder array. The PMMA components of the cylinder cores were selectively removed by wet etching. The physical deposition of a metal layer (Ag, $\mathrm{Au}, \mathrm{Cu}$, or Pt) and the subsequent lift-off of block copolymer template generated a hexagonal metal nanoparticle array on the transparent pyrex substrate. The nanoparticle diameter $(\sim 40 \mathrm{~nm})$ and the center-to-center distances between neighbouring nanoparticles $(\sim 80 \mathrm{~nm})$ were consistent with the periodic self-assembled morphology of the block copolymer template. For antibody detection, the resulting plasmonic nanoparticle array was sequentially modified with a carboxylic acid terminated selfassembled monolayer and a prostate specific antigen (PSA) at the nanoparticle surface. The following highly specific binding events between the antigen (PSA) and antibody (anti-PSA) were monitored by UV-vis spectrum measurement. Since PSA is a wellknown biomarker for prostate cancer, the ultrasensitive detection technologies for PSA or anti-PSA might provide an opportunity for the early diagnosis of prostate cancer. As a model assay for prostate cancer diagnosis, our plasmonic sensor was designed to detect anti-PSA that specifically binds with PSA. ${ }^{10,32,33}$

Fig. 3 presents scanning electron microscopy (SEM) images of Ag nanoparticle arrays having different nanoparticle height $(h)$ prepared by block copolymer lithography. The insets display tilted cross-sectional views of nanoparticle arrays. When the deposited metal thickness was insufficient $(h=5 \mathrm{~nm})$, metal nanoparticles showed highly irregular shapes (Fig. 3(a)). However, as $h$ increased from 10 to $30 \mathrm{~nm}$, the geometry of nanoparticles gradually became conical-shape (Fig. 3(b) and (c)). This conical-shape was caused by the gradual metal accumulation at the entrance of the template nanopores during metal deposition (Fig. 3(d)).
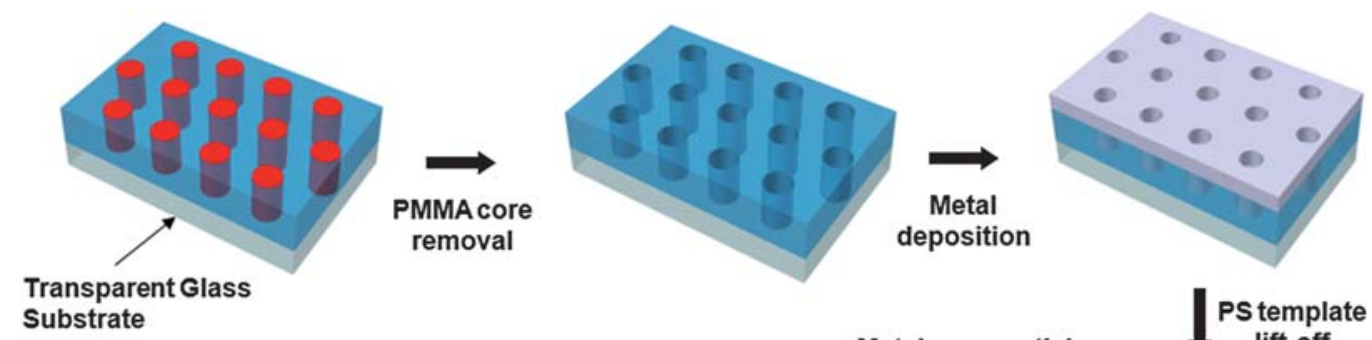

Substrate
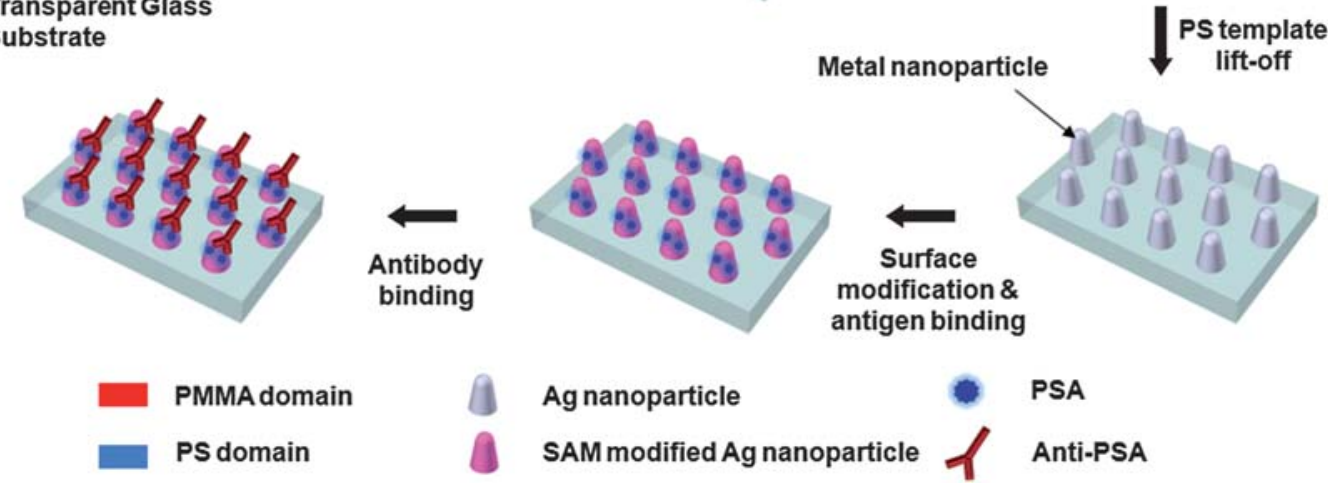

Fig. 2 A schematic illustration of LSPR biosensing employing block copolymer lithography. A hexagonal nanoporous template was prepared on a transparent pyrex substrate by block copolymer lithography. The Ag nanoparticle array replicating block copolymer template morphology was fabricated by thermal evaporation and lift-off. The nanoparticle array was modified with a SAM layer for antigen binding. The covalent binding of the antigen and the subsequent specific binding between the antigen and antibody were monitored by the UV-vis spectrum. 


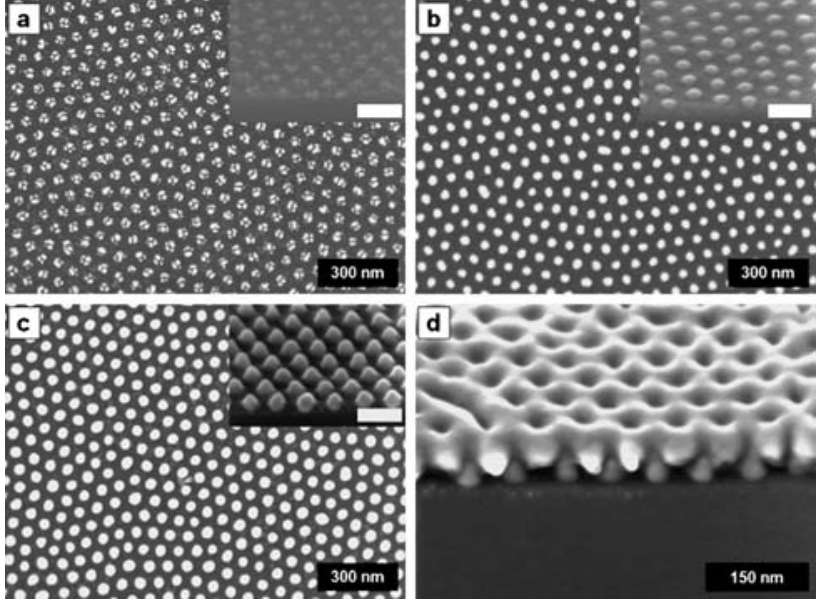

Fig. 3 SEM images of Ag nanoparticle arrays with various particle heights of (a) $5 \mathrm{~nm}$, (b) $10 \mathrm{~nm}$ and (c) $30 \mathrm{~nm}$. Note that the nanoparticle gradually became conical-shaped, as the height increased. The insets show cross-sectional SEM images of Ag nanoparticle arrays. White scale bars correspond to $100 \mathrm{~nm}$. (d) A cross-sectional SEM image of Ag nanoparticles before lifting-off block copolymer nanotemplate.

Fig. 4 shows the UV-vis absorbance spectra of the various noble metal nanoparticle arrays prepared by block copolymer lithography. Despite the same morphology of nanoparticle arrays, the LSPR $\lambda_{\max }$ of $\mathrm{Cu}, \mathrm{Au}, \mathrm{Ag}$, and Pt were located at various wavelengths of $613,567,452$, and $468 \mathrm{~nm}$, respectively. The Ag nanoparticle array was found to exhibit the most intense and sharpest absorbance band, which was appropriate for sensing applications. In contrast, Pt exhibited an extremely broad and weak absorbance band. ${ }^{37}$

Fig. 5(a) presents the UV-vis absorbance spectra of $\mathrm{Ag}$ nanoparticle arrays with $5 \mathrm{~nm}<h<40 \mathrm{~nm}$. The nanoparticle array with $h=30 \mathrm{~nm}$ shows the most intense absorbance peak.

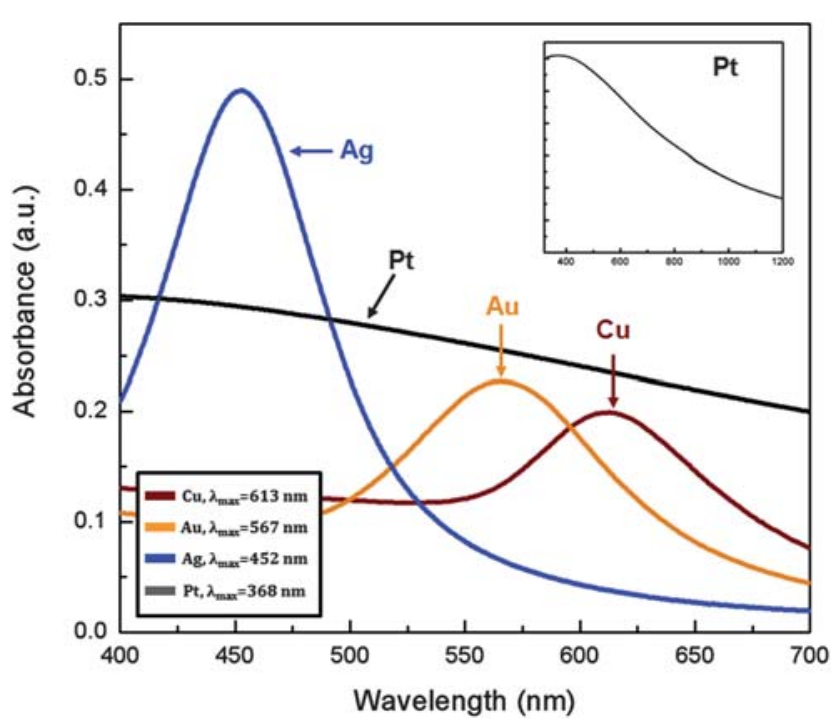

Fig. 4 UV-vis absorbance spectra of various noble metal nanoparticle arrays having the same geometry (nanoparticle diameter: $40 \mathrm{~nm}$, height: $30 \mathrm{~nm}$ ). The inset shows the extremely broad and weak absorbance band of Pt throughout the UV-vis region.
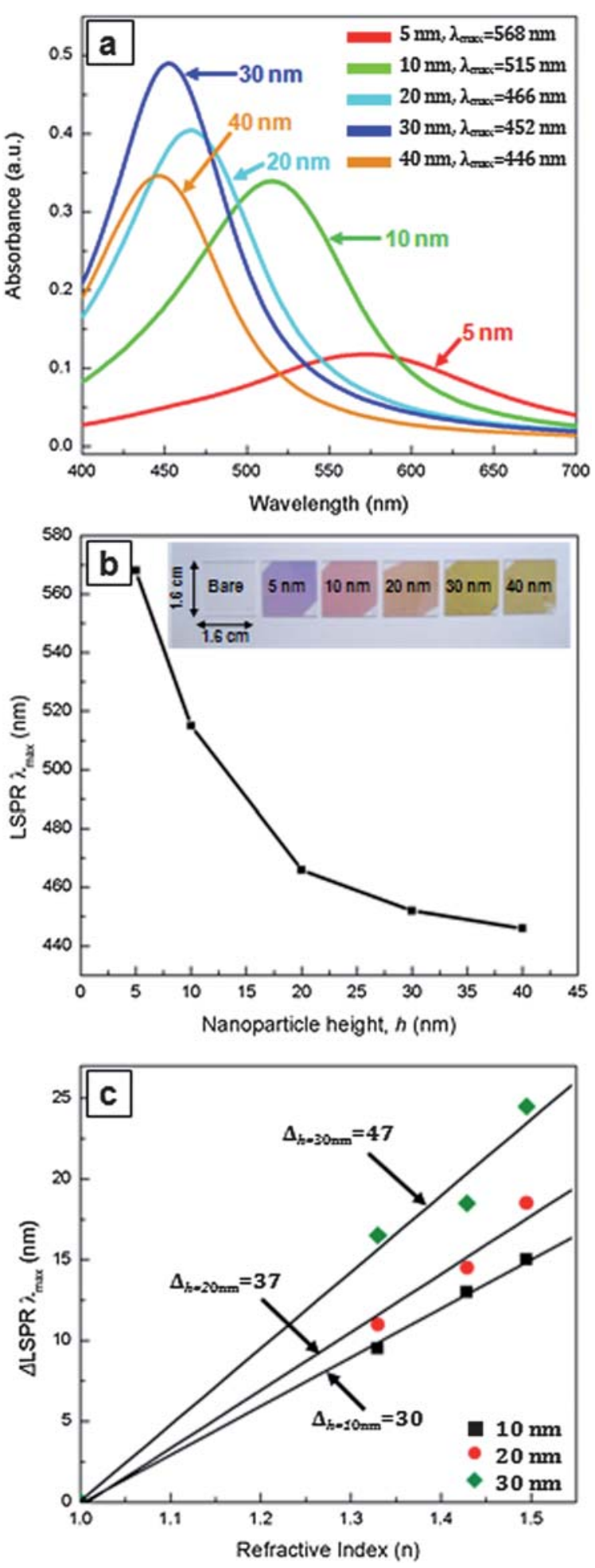

Fig. 5 (a) UV-vis absorbance spectra of Ag nanoparticle arrays with various heights from 5 to $40 \mathrm{~nm}$. (b) LSPR $\lambda_{\max }$ variation as a function of nanoparticle height. The inset is a photograph of Ag nanoparticle array chips $(1.6 \mathrm{~cm} \times 1.6 \mathrm{~cm}$ size) with various nanoparticle heights. (c) LSPR sensitivity $\left(\Delta \lambda_{\max }\right)$ in response to the refractive index of dielectric surrounding.

Fig. 5(b) presents the LSPR $\lambda_{\max }$ plotted against $h$. The absorption band underwent blue-shift as a function of $h{ }^{38}$ For a given height change, the blue shift was large when the 
nanoparticle height was small. This trend is consistent with the theoretical prediction for plasmonic behavior of gold triangular prisms. ${ }^{39}$ The inset image shows the photograph of Ag plasmonic arrays prepared on $1.6 \times 1.6 \mathrm{~cm}^{2}$ sized glass chips. While a bare glass substrate did not produce any optical signal, Ag plasmonic arrays with different nanoparticle heights demonstrated a pronounced color variation. In order to verify the sensing capability of a plasmonic structure, it is convenient to measure the variation of LSPR $\lambda_{\max }$ in accordance with the refractive index of the surrounding medium. Fig. 5(c) shows the LSPR $\lambda_{\max }$ variation of $\mathrm{Ag}$ nanoparticle arrays immersed in three solvents with different refractive indices (water: $\eta=1.33$, EtOH/Toluene: $\eta=1.429$, Toluene: $\eta=1.495)$. The LSPR $\lambda_{\max }$ shift $\left(\Delta \lambda_{\max }\right)$ was roughly proportional to the refractive index of the surrounding solvent. From a linear fit, the sensitivity factor, $\Delta \lambda_{\max } / \mathrm{RIU}$, could be found, which is defined as the relative changes in resonance wavelength with respect to the refractive index change of the surrounding medium. (ESI, $\uparrow$ Fig. S2) The sensitivity factors were determined to be $30 \mathrm{~nm} / \mathrm{RIU}$ for $h=10 \mathrm{~nm}, 37 \mathrm{~nm} /$ RIU for $h=20 \mathrm{~nm}$, and $47 \mathrm{~nm} / \mathrm{RIU}$ for $h=30 \mathrm{~nm}$, respectively.

In this work, we have investigated the detection of a specific antibody (anti-PSA) by means of a plasmonic array chip selectively decorated with an antigen (PSA). This approach is useful to search for specific interactions between antigens and antibodies and provides valuable information to understand specific diseases such as Alzheimer's. ${ }^{10}$ The sequential immobilization of the SAM, antigen, and antibody at the nanoparticle surface of a plasmonic array is illustrated in Fig. 6(a). The nanoparticle array possessing the highest LSPR sensitivity ( $D=40 \mathrm{~nm}, h=30$ $\mathrm{nm})$ was used in this analysis. Each immobilization step monitored by UV-vis absorption measurement demonstrated gradual red-shift of LSPR $\lambda_{\max }$, as presented in Fig. 6(b) and 6(c). For antibody detection, Ag nanoparticle surfaces were selectively modified with a mixture SAM consisting of $3: 11 \mathrm{mM} \mathrm{OT} / 1 \mathrm{mM}$ 11-MUA and aqueous antigen solution containing $1 \mu \mathrm{g} \mathrm{m} \mathrm{m}^{-1}$ PSA with $20 \mu \mathrm{g} \mathrm{ml}^{-1}$ EDC. The PSA antigen molecules were covalently bound to the metal nanoparticle surface through amide bonding. In the final antibody detection step, the $\Delta \lambda_{\max }$ was crucially dependent on the concentration of antibody solution. The incubation in $10 \mathrm{ng} \mathrm{ml}^{-1}$ anti-PSA solution for $1 \mathrm{~h}$ caused $\Delta \lambda_{\max }$ of $+4 \mathrm{~nm}$, while that in $10 \mu \mathrm{g} \mathrm{ml}^{-1}$ solution raised the $\Delta \lambda_{\max }$ of $+19 \mathrm{~nm}$.

Fig. 6(e) and 6(f) show the AFM images and the corresponding height profiles of the nanoparticle arrays solely modified with SAM (Fig. 6(e)) and sequentially immobilized with SAM, PSA, and anti-PSA (Fig. 6(f)), respectively. The height profiles show that the average height of SAM modified nanoparticles was 18.24 $\pm 1.3 \mathrm{~nm}$, which increased up to $22.18 \pm 1.5 \mathrm{~nm}$ ( $\sim 4 \mathrm{~nm}$ increase) after the antigen and antibody immobilization.

Fig. 7 shows control experiments revealing that the $\Delta \lambda_{\max }$ is negligible without specific binding between antigen and antibody. When the 'bare' Ag nanoparticle array chip was incubated
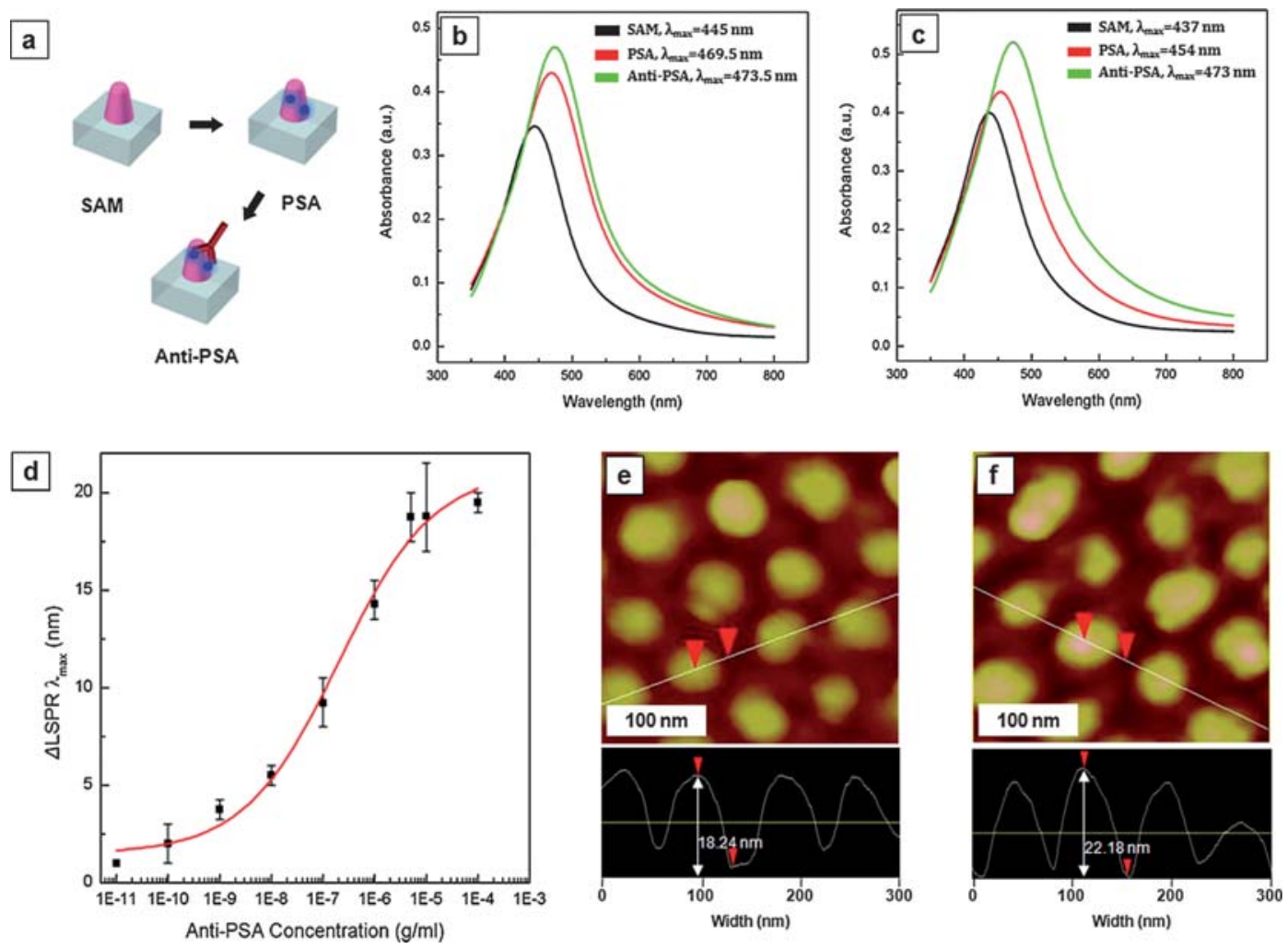

Fig. 6 (a) A schematic description of the sequential immobilization of SAM, antigen, and antibody on a plasmonic array. Absorption spectra variations for (b) low $\left(10 \mathrm{ng} \mathrm{ml}^{-1}\right)$ and (c) high concentration $\left(10 \mu \mathrm{g} \mathrm{ml}^{-1}\right)$ anti-PSA detection. (d) the magnitude of $\Delta \lambda_{\max }$ plotted against anti-PSA solution concentration $\left(10 \mathrm{pg} \mathrm{ml}^{-1}\right.$ to $\left.100 \mu \mathrm{g} \mathrm{ml}^{-1}\right)$. AFM images and height profiles of Ag plasmonic arrays modified either (e) solely with SAM (average height $=$ $18.24 \pm 1.3 \mathrm{~nm}$ ) or (f) sequentially with SAM, PSA and anti-PSA (average height $=22.18 \pm 1.5 \mathrm{~nm}$ ). 

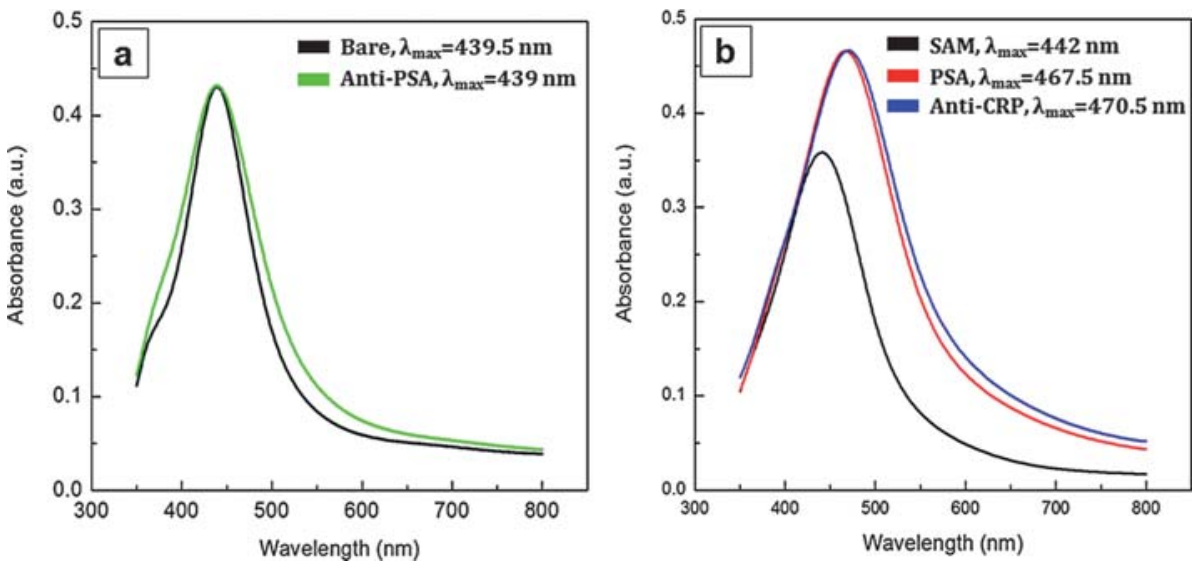

Fig. 7 (a) LSPR absorbance spectra of the 'bare' Ag nanoparticle array chip incubated in $10 \mu \mathrm{g} \mathrm{ml}^{-1}$ anti-PSA solution with $20 \mu \mathrm{g}$ ml ${ }^{-1}$ EDC for $1 \mathrm{~h}$ followed by washing with PBS and DI water. (b) LSPR absorbance spectra for the control experiment that nonbinding anti-C-reactive protein (CRP) was used.

in $10 \mu \mathrm{g} \mathrm{ml}^{-1}$ anti-PSA solution with $20 \mu \mathrm{g} \mathrm{ml}^{-1} \mathrm{EDC}$ for $1 \mathrm{~h}$, no noticeable $\Delta \lambda_{\max }$ was observed without SAM and PSA layers (Fig. 7(a)). For Fig. 7(b), the Ag nanoparticle array chip was first incubated in $1 \mu \mathrm{g} \mathrm{ml}^{-1}$ PSA (TCN solution) with EDC (PBS) for $1 \mathrm{~h}$. Further incubation in $10 \mu \mathrm{g} \mathrm{ml}^{-1}$ anti-CRP(PBS) for $1 \mathrm{~h}$ caused no noticeable $\Delta \lambda_{\max }$ without specific binding.

\section{Conclusions}

We have demonstrated a novel strategy for creating a highly dense and uniform plasmonic biosensor array by block copolymer lithography. A scalable and parallel block copolymer lithography employing a small molecular weight block copolymer as an assembly promoter generated a hexagonal conicalshape metal nanoparticle array on a large-area transparent glass substrate. The resulting plasmonic array displayed a UV-vis absorbance band sufficiently narrow and intense for biosensing. The specific binding of the antibody with the antigen that occurred at the plasmonic metal particle surface raised the pronounced peak shift of an absorption band. A remarkably low detection limit of $0.1 \sim 1 \mathrm{ng} \mathrm{ml}^{-1}$ that satisfies the requirement for a prostate cancer sensor has been established in this label-free sensing system. Our low-cost, large-scale fabrication of plasmonic nanostructure exploiting block copolymer lithography is potentially useful for various photonic and optoelectronic devices.

\section{Acknowledgements}

This work was supported by National Research Laboratory Program (R0A-2008-000-20057-0), the National Research Foundation (NRF) grant (2008-0062204, 2009-0064787, 20090069888), World Class University (WCU) program (R32-2008000-10051-0), Platform Project grant (10033636-2009-11) funded by the Korean government.

\section{Notes and references}

1 J. P. Krenn, Nat. Mater., 2003, 2, 210.

2 S. A. Maier, P. G. Kik, H. A. Atwater, S. Meltzer, E. Harel, B. E. Koel and A. A. G. Requicha, Nat. Mater., 2003, 2, 229.
3 W. L. Barnes, A. Dereux and T. W. Ebbesen, Nature, 2003, 424, 824. 4 S. Nie and S. R. Emory, Science, 1997, 275, 1102.

5 A. M. Michaels, M. Nirmal and L. E. Brus, J. Am. Chem. Soc., 1999, 121, 9932.

6 G. C. Schatz, Acc. Chem. Res., 1984, 17, 370.

7 S. Kim, N. Cheng, J.-R. Jeong, S.-G. Jang, S.-M. Yang and W. T. S. Huck, Chem. Commun., 2008, (31), 3666.

8 R. Elghanian, J. J. Storhoff, R. C. Mucic, R. L. Letsinger and C. A. Mirkin, Science, 1997, 277, 1078.

9 G. Bauer, F. Pittner and Th. Schalkhammer, Mikrochim. Acta, 1999, 131, 107.

10 A. J. Haes, W. P. Hall, L. Chang, W. L. Klein and R. P. Van Duyne, Nano Lett., 2004, 4, 1029.

11 H. M. Hiep, T. Endo, M. Saito, M. Chikae, D. K. Kim, S. Yamamura, Y. Takakmura and E. Tamiya, Anal. Chem., 2008, 80, 1859.

12 C. F. Bohren, D. R. Huffman, Absorption and scattering of light by small particles; John Wiley \& Sons: New York 1983.

13 K. A. Willlets and R. P. Van Duyne, Annu. Rev. Phys. Chem., 2007, 58, 267.

14 T. R. Jensen, M. D. Malinsky, C. L. Haynes and R. P. Van Duyne, J. Phys. Chem. B, 2000, 104, 10549.

15 E. M. Hicks, S. Zou, G. C. Schatz, K. G. Spears and R. P. Van Duyne, Nano Lett., 2005, 5, 1065.

16 D. Wouters and U. S. Schubert, Angew. Chem., Int. Ed., 2004, 43, 2480.

17 S. Y. Chou, P. R. Krauss and P. J. Renstrom, Science, 1996, 272, 85.

18 A. J. Haes, L. Chang, W. L. Kleing and R. P. Van Duyne, J. Am. Chem. Soc., 2005, 127, 2264.

19 M. Park, C. Harrison, P. M. Chaikin, R. A. Register and D. H. Adamson, Science, 1997, 276, 1401.

20 T. Thurn-Albrecht, J. Schotter, G. A. Kastle, N. Emley, T. Shibauchi, L. Krusin-Elbaum, K. Guarini, C. T. Black, M. T. Tuominen and T. P. Russell, Science, 2000, 290, 2126.

21 S. O. Kim, H. H. Solak, M. P. Stoykovich, N. J. Ferrier, J. J. de Pablo and P. F. Nealey, Nature, 2003, 424, 411.

22 S. O. Kim, B. H. Kim, D. Meng, D. O. Shin, C. M. Koo, H. H. Solak and Q. Wang, Adv. Mater., 2007, 19, 3271.

23 I. Bita, J. K. W. Yang, Y. S. Jung, C. A. Ross, E. L. Thomas and K. K. Berggren, Science, 2008, 321, 939.

24 C. Tang, E. M. Lennon, G. H. Fredrickson, E. J. Kramer and C. J. Hawker, Science, 2008, 322, 429.

25 B. H. Kim, D. O. Shin, S.-J. Jeong, C. M. Koo, S. C. Jeon, W. J. Hwang, S. Lee, M. G. Lee and S. O. Kim, Adv. Mater., 2008, 20, 2303.

26 D. H. Lee, W. J. Lee and S. O. Kim, Nano Lett., 2009, 9, 1427.

27 S.-J. Jeong, J. E. Kim, H.-S. Moon, B. H. Kim, S. M. Kim, J.-B. Kim and S. O. Kim, Nano Lett., 2009, 9, 2300.

28 D. O. Shin, B. H. Kim, J. H. Kang, S.-J. Jeong, S. H. Park, Y.-H. Lee and S. O. Kim, Macromolecules, 2009, 42, 1189. 
29 C. T. Black, R. Ruiz, G. Breyta, J. Y. Cheng, M. E. Colburn, K. W. Guarini, H.-C. Kim and Y. Zhang, IBM J. Res. Dev., 2007, 51, 605.

30 Y. S. Jung, W. J. Jung and C. A. Ross, Nano Lett., 2008, 8, 2975.

31 S.-J. Jeong, G. Xia, B. H. Kim, D. O. Shin, S.-W. Kang and S. O. Kim, Adv. Mater., 2008, 20, 1898.

32 G. Zheng, F. Patolasky, Y. Cui, W. C. Wang and C. M. Lieber, Nat. Biotechnol., 2005, 23, 1294.

33 H. B. Carter, N. Engl. J. Med., 2004, 350, 2292.

34 P. Mansky, Y. Liu, E. Huang, T. P. Russell and C. Hawker, Science, 1997, 275, 1458.
35 T. Xu, H.-C. Kim, J. DeRouchey, C. Seney, C. Levesque, P. Martin, C. M. Stafford and T. P. Russell, Polymer, 2001, 42, 9091.

36 S. Ji, G. Liu, F. Zheng, G. S. W. Craig, F. J. Himpsel and P. F. Nealey, Adv. Mater., 2008, 20, 3054.

37 C. Langhammer, Z. Yuan, I. Zorić and B. Kasemo, Nano Lett., 2006, 6, 833.

38 G. H. Chan, J. Zhao, E. H. Hicks, G. C. Schatz and R. P. Van Duyne, Nano Lett., 2007, 7, 1947.

39 K. K. Shuford, M. A. Ratner and G. C. Schatz, J. Chem. Phys., 2005, 123, 114713 